DOI https://doi.org/10.32841/2409-1154.2019.40.3.9

\author{
Rasulov Rustam Kamal, \\ Doktor der Philologie, Professor \\ Aserbaidschanische staatliche Universität für Erdöl und Industrie
}

\title{
„DAS BUCH DEDE KORKUT“ UND DAS „NIBELUNGENLIED“: DARSTELLUNG DER LITERARISCHEN PORTRÄTS
}

\begin{abstract}
Анотація. У німецькій та турецькій літературі Середньовіччя методи дослідження людства та їхні принципи відіграють важливу роль у дослідженні поетичних систем. Автори Середньовіччя зосередили увагу на способах зображення портрета, які змінювались за жанром.

«Книга Деде Коркут» та «Нібелунглієд» аналізують складні портрети на типологічному та порівняльному рівні. Наше дослідження можна розділити на п'ять портретних груп на основі таких критеріїв:

1. Ступінь узагальнення уявлення (одиночний та груповий портрет).

2. Тема презентації: представлена частина зовнішності людини.

3. Метод викладу літературної фігури: зображений портрет, портрет - гендерна рівність, портрет-порівняння тощо.

4. Ступінь етикету презентації.

5. Характер введення портрета в літературну дію.

В епосі «Книга Деде Коркут» важливу роль відіграє метод літературного викладу, портретний метод.

У структурі епічного тексту узагальнені «портретні тексти», які утворені епітетом. Крім чеснот епічних героїв, у «портретних текстах» представлені психологія їхнього характеру, їхня зовнішність та символи війни (кінь, обладунки, зброя). Ці портрети також визначають становище огузької знаті (бейсів) у соціальній ієрархії:
\end{abstract}

Ключові слова: епос, сюжет, герой, драма, «Книга Деде Коркут» та «Нібелунгенлід».

Ghaflet Kodschas Sohn Scher Schemseddin, der ohne Erlaubnis von Bajindur Chan dessen Feinde überfallen hatte, der sechzigtausend Ungläubige hatte Blut spucken lassen, der den Schnee auf den Mähen seines Apfelschimmels liegen ließ.

Beirek mit dem grauen Pferd, derjenige, der wie ein Blitz aus Parasars Burg Baiburd geflogen kam und vor seinem bunten Brautzelt erschien, Hoffnung von sieben Mädchen Liebling der Oghusen, Herr Kasans Vertrauensmann.

Kasilik Kodschas Sohn Herr Jegenk, derjenige, der mit der vornehmen Erscheinung, wenn er einen scharf ansah, mutig wie der grauschwarze Adler, mit seinem reich verzierten Gürtel und mit goldenen Ohrhängern und derjenige, der die Herren der strammen Oghusen einen nach dem andern aus Sattel hebeln konnte.

Kasans Oheim Arus Kodscha mit dem Pferdemaul, derjenige, dessen Fußgelenke nicht bedeckt wurden von einem mantel aus dem Fell von sechzig Widdern, dessen Ohren nicht bedeckt wurden, wenn er eine Mütze aus dem Fell von sechs Widdern trug, mit Oberschenkel lang wie ein Querbalken du mit mageren Waden.

Bügdüz Emen mit dem blutigen Schnurrbart, der das Antlitz des Propheten gesehen hatte und der nach seiner Heimkehr dessen Vertreter bei den Oghusen war, aus dessen Schnurrbart bei einem
Wutausbruch Blut tropfte, kam angaloppiert und sagte: „Schwinge deinen Säbel, mein Herr Kasan, da bin ic!“

Ejlik Kodschas Sohn Alp Eren, der die Ungläubigen mit mehr Verachtung behandelte als Hunde, der sein Land verließ und sein Pferd über den Fluss des Zuschauenden Hengsten schwimmen ließ, der Tscheschme, Tochter des Weißen Königs heiratete, der Sufi Mälik Blut spuken ließ, der sich in vierzig Mantel hüllte und die Schönen Töchter von siebenunddreißig Burgherren stahl, der sie eine nach der anderen umarmte und auf das Gesicht und Lippen küsste! [1, p. 49].

In diesen Porträts werden sowohl der Erscheinungsbild, wie auch die Persönlichkeit und innere Charakterzüge zum Ausdruck gebracht [3, p. 36].

Diese Porträts, die mit Hilfe der Volksausdrücke und der Sprache Ozan gebildet wurden, sind mit dem Verhalten und der Tätigkeit der literarischen Gestalten verbunden. Sie spiegeln dadurch deren außerordentlichen heroischen Merkmale wider. In machen Fällen werden die Porträts so übertrieben dargestellt, dass man die realen Personen wie die Fremde, mythische Gestalten, die sonst nur in Märchen vorkommen, wahrnimmt. Dennoch sind sie aufgrund ihres Verhaltens und ihrer Tätigkeit reale Menschen [4, p. 61].

Kasans Oheim Arus Kodscha wird zum Beispiel folgenderweise dargestellt: „Derjenige, dessen Fußgelenke nicht bedeckt wurden von einem Mantel aus dem Fell von sechzig Widdern, dessen Ohren nicht bedeckt wurden, wenn er eine Mütze aus dem Fell von sechs Widdern trug, mit Oberschenkeln lang wie ein Querbalken und mit mageren Waden" [1, p. 49].

In dem Porträt von Kasans Oheim Arus Kodscha beispielweise ist deutlich zu sehen, dass ein Mensch, ,dessen Fußgelenke nicht bedeckt wurden von einem Mantel aus dem Fell von sechzig Widdern", übernatürlich groß sein musste.

Kara Göne, Emen, der Hirte Karadschuk, Jegenek und die anderen Helden werden ebenfalls als kräftige und große Menschen dargestellt.

Das sind die Eigenschaften, die den physischen Kräften der Helden entsprechen. Die Entsprechungen ihrer inneren Kräfte mit den äußeren Merkmalen sind als eine aufs Engste verbundene Einheit zu betrachten. Herr Kasan zum Beispiel ,wirft einen vom Berg abgesplitterten Felsen mit seiner Brust zur Seite weg und zerreißt die an seine Hände und Arme gebundene dünne Schnur“.

Der Hirte Karadschuk besaß ,eine Schleuder, deren Leder, aus der Haut eines dreijährigen Kalbes gegerbt war. Der Griff war aus Harren von drei Ziegen und die Gabel aus dem Haar einer Ziege. In einem Schuss schleuderte er einen zwölf Batman schweren Stein“. Dieser Hirte riss sogar Bäume samt Wurzeln heraus. Durch diese Beschreibungen wird die physische Stärke des Hirten Karadschuk auf metonyme Weise zum Ausdruck gebracht. 
Über Kara Göne wird Folgendes erzählt: „Herrn Kasans Bruder Kara Göne, derjenige, den der Herr in die Welt gesetzt hatte bei der Mündung des Schwarzen Baches, dessen Wiege mit der Haut eines schwarzen Stieres bedeckt war, der in Wut entbrannt, einen schweren Stein zur Asche schlug, der sich einen Schnurrbart in sieben Strähnen im Nacken zusammenband“.

Hiermit wird äußerliche Größe von Kara Göne (sich einen Schnurrbart in sieben Strähnen im Nacken zusammenbinden) mit der Psychologie seiner Welt und seiner Expressivität (in Wut entbrennen) als Einheit dargestellt.

In diesen Porträttexten lässt sich die einheitliche Erscheinung des Porträts mit der Psychologie des Merkmals in der Gestalt von Emen deutlich erkennen, der als ,der blutige Schnurrbart das Antlitz des Propheten gesehen hatte und der nach seiner Heimkehr dessen Vertreter bei den Oghusen war. „Dieser kurzer Ausdruck lässt uns die Psychologie seines Charakters und sein äußeres Bild - ,aus dessen Schnurrbart bei einem Wutausbruch Blut tropfte" - klar und deutlich vorstellen.

Im „Buch des Dede Korkut“ werden neben oghusischen Helden auch die Porträts der ungläubigen Helden hyperbolisch dargestellt, wie z.B. die Person des Arschuns Sohn Fürsten Direk, des Burgherrn von Düsmürd, der ,sechzig Ellen lang war und sechzig Batman schwere Keule schwang" [1, p. 49].

Diese Gestalten aus einer anderen Welt sehen äußerlich anders und unbestimmt aus. Sie werden entweder nicht rechtzeitig erkannt, oder sie sind leicht zu übersehen. Asrael bzw. Gabriel wird in der islamischen Mythologie als mythische Gestalt betrachtet. Daher war Deli Domrul zu Tode erschreckt, als er Gabriel vor sich sah:

Mein Mund ist von innen kalt wie Eis,

meine Gebeine sind in Salz verwandelt.

$\mathrm{Du}$, Greis mit deinem weißen Bart,

Greis mit deinen trüben Augen,

komm, sage mir, was für ein schrecklicher Greis bist du? $[1$, p. 80]

Obwohl die literarischen Gestalten der Ungläubigen körperlich stark und ihr Aussehen schrecklich und angstflößend dargestellt werden, sind sie hinsichtlcih ihres Glubens und des Geistes schwächer als die oghusischen Helden.

Ein paar Bekleidungsdetailis sind gebräuchliche Mittel zur Beschreibung des Erscheinungsbildes der Gestalten. Die Krieger des Königs Schökli, z.B. tragen Kriegshelme und Rüstung und „,hre Haare sind in Tonsur geschnitten“. Die oghusischen Krieger dagegen haben eine vornehme Erscheinung. Sie haben ein Tuch um den Rücken und einen Kaftan an. Der Hirte Karadschuk trägt eine Mütze. Helden wie Emen und Jegenek tragen goldene Ohrhänger.

Diese Merkmale verhelfen uns ein klares Bild über das Aussehen der dazugehörigen Gestalten zu erhalten.

Die oghusischen Helden sind körperlich stark und von „furchterregender Statur". Ihr innerer Charakter sowie ihr Erscheinungsbild werden - metaphorisch betrachtet - mit den anderen Wesen der Natur, den Tieren, gleichgestellt. Herr Kasan und Bajindur Chan werden als Panther (Leopard) betrachtet. Als Arus Kodscha wird mit dem Epitheton „der Mensch mit dem Pferdemaul" beschrieben, die Mädchen als Vögel usw. Das alles ist auf die Existenz der Legenden und Mythen über Oghusen im Volksmunde zurückzuführen.

Die ethnographisch-archäologischen Quellen liefern uns Belege dafür, dass die Oghusen tatsächlich körperlich von großer Statur waren.
Das Porträt des mit der Löwenmilch Basat wird folgenderweise dargestellt: „Mein Herrin, aus dem Schilffeld kommt ein Löwe und reißt Pferde, aber seine wiegende Gangart ist eher die eines Menschen. Er greift die Pferde und schlürft ihr Blut" [1, p. 98].

In der Gegenüberstellung von Natur und Zivilsation symbolisiert der Löwe die Natur. Basat wendet sich von der Natur ab und wendet sich den Menschen zu, kehrt, zur Zivilsation zurück. Und der Dede Korkut versucht, ihn in die Menschengesellschaft zu intengrieren, indem er ihm einen Namen, den Namen "Basat" gibt. Basat wird zum Bestandteil der Gesellschaft der Gesellschaft und widersetzt sich bald seiner alten Weelt, der natur, die ihn großgezogen hatte, und nicht hatte eingehen lassen [5, p. 16].

Herr Kasan versucht, seinen unerfahrenen Sohn das Porträt der Feindestruppen auf vergleichender Weise zu übermitteln:

Komm her, mein Sohn, mein löwenhafter Sohn.

Was daherkommt, brausend wie das (Schwarze) Meer, ist das

Herr der Ungläubigen.

Was daherkommt, flammend wie die Sonne, sind die Helme, Die sie tragen.

Was daherkommt, leuchtend wie die Sterne, sind ihre Lanzen.

Es sind die ungläubigen Feinde mit ihren Irrglauben [1, p. 170].

Nach Kamal Abdulla gehören solche Ausdrücke ("wie das Meer", "Wie die Sonne", "wie die Sterne") dem Mythos-Prozess des Vergleichens an. Anderes gesagt, es ist ein Blick ins Vergleichen aus der Perspektive des Mythos [5, p. 183].

In verschiedenen Textstellen des "Buch des Dede Korkut" werden die Ungläubigen oft mit "(Kolk) Raben" und "von Tollwut befallenen Tieren" verglichen:

"Sechszehntausend schwarztrockige Ungläubige stiegen auf ihre Pferde. Sie ritten schnurstarks zu Kasan. Dort sahen sie, wie sechs Staubwolken hinuntenwehten. Einige sagten: ,Der Staub stammt von Hirschen.' Andere sagten: ,Der Staub stammt vom Feind'. Kasan sagte: ,Wäre es der Staub von Hirschen, es wäre eine Wolke, oder höchstens zwei. Was dort daherkommt, ist der Feind, das sollt ihr wissen'. Der Staub teilte sich und etwas leuchtete auf die Sonne, etwas brauste wie das Meer und etwas schien schwarz wie der Wald. Sechszehntausend Ungläubige, in Hemden aus grobem Faden, mit Hüten aus Ziegenhaar, mit ihrem Irrgaluben und ihrer hitzigen Sprache tauchten vor Kasan auf".

In diesem Kontext sind die Ausdrücke wie "quzğun" [,Kolkraben“] und „,quzğun dili" [,Sprache der Kolkraben“ oder ,ihre hitzige Sprache „] typisch für die Welt der Ungläubigen. Der Rabe (,[Kasan] sah, wie Raben herumflogen und Jagdhunde herumsaßen, dort, wo sein Zelt stand) tritt als eines der drei Gesichter des Schicksalsrades auf. Seien Funktion ähnelt der des Falken. Vermittelt uns die die phonetische Motivation von Wörtern wie ,azğun”[,wild“] und ,quzğun” [,Kolkrabe“] die Bedeutung „blutgieriges Raubtier“, wird das Wort „quzğun dili” [,,Sprache der Kolkraben“] mit dem Motiv „Lärm, Gegröle“ assoziiert. Diese Assoziation ermöglicht es uns als wichtiges Detail, das Porträt der Ungläubigen zu erfassen. „,quzğun dili” [,,Sprache der Kolkraben“] ist das substanzielle Attribut der Schicksalsgestallten.

In der Regel werden die Körper der Helden im „Buch des Dede Korkut" nicht durch Metamorphosen beeinflusst. Aber diese Regelmäßigkeit kennt auch ein paar Ausnahmefälle. Die Metamorphose bezieht sich nicht auf die Haupthelden, sondern ausschließlich auf die Gestalten, die uns fremd erscheinen, d.h. auf Gestalten wie Feind und Ungläubiger: ,[Deli Domrul] zückte seinen Säbel aus 
dunklem Stahl und griff Asrael an. Asrael verwandelte sich in eine Taube und flogt durch das Fenster davon" [1, p. 80].

In der archetypischen Gesellschaft der Oghusen (in der Weltordnung der Oghusen), die auf dem Phallozentrismus, der Phallokratie und dem Logozentrismus beruhte, wird sich das männliche Schönheitsideal der oghusichen Adeligen durch die grauen Haare definiert. Deren schönes Antlitz wird als ästhetischen Maß anerkannt. Das Verständnis der epischen Welt war von der heroischen (männlichen) Warte aus ein Mittel für die Existenz der epischen Sprache.

„Als er [Egrek] sich umschaute, sah er einen braunäugigen jungen Krieger, schön wie der Mond in der vierzehnten Nacht. Er schlief mit Schweißperlen auf der Stirn“" [1, p. 113].

Bei den Helden der Oghusen bildet die moralische und innerliche Schönheit (schönes Aussehen und Intelligenz) eine Einheit. Sie sind diejenigen, die schön aussehen und intelligenz sind. Diese Schönheit spiegelt sich in dem Epos sogar auf magischer Weise wider: "Unter den Oghusen gab es vier Männer, die verschleiert umherliefen. Außer Kanturali waren dies Kara Tschekür, dessen Sohn Kirk Kinuk und Beirek mit dem grauen Pferd" [1, p. 87].

Die Farbe an sich ist im emprischen Text neutral. Nur aus der Perspektive des Barden [Ozan] heraus wird ihr eine Bedeutung zugeschrieben. Das Erkennen dieser Perspektive ist von den Werten in den stereotypischen Systemen abhängig und mit der ontologisch-ästhetischen Erfahrung des Betrachters verbunden. Diese Vorgehensweise mag für die gesamte mittelalterliche Weltanschauung und Denkweise gegolten haben. In der islamisch-geprägten Denkweise und dem epischen Verständnis der Oghusen spielten zwei Farben, Weiß und Schwarz, eine besondere Rolle. Neben den gemeinsamen Merkmalen ersetzten sich diese Farben symbolisch gegenseitig. Beide Farben waren bei der mittelalterlichen Wahrnehmung der Umwelt von ausschlaggebender Bedeutung. Während Weiß in der mentalen Kultur und Denkweise der Oghusen mit dem Licht, der Schönheit und der Aktivität des Geistes zusammenhing, bedeutete das Schwarz hingegen Finsternis, Hässlichkeit, Widerwärtigkeit und Sünde. Dieser Kontrast der Farben verdeutlicht uns ebenfalls den Konflikt zwischen dem Guten und dem Bösen.

„Siebentausend Ungläubige mit einem Schlitz hinten in ihren Kaftanen, ihre Haare in Tonsur geschnitten, von faulen Glauben, Feinde des Islam, bestiegen ihre Gäule und galoppierten".

Der König von Trapezunt wählte für die Suche nach Kanturali und Seldschan ,sechshundert Ungläubige [aus], mit Kettenhemden aus blaueen Stahl über ihren schwarzen Kleidern“" [1, p. 90].

Einer von denen, die uns fremd erscheinen mögen, ist Jaltadschuk, Sohn des Lügners. Er verbreitet die Nachricht, dass Beirek tot ist. Damit möchte er erreichen, Banu Tschitschek, die Verlobte des Beirek zu heiraten.

Die Innen-Oghusen scheinen dieser Nachricht nicht zu glauben. Wegen seiner Lügenschichten benennt man ihn abscheulich Jaltadschuk, Sohn des Lügners. In der lebendigen und gesprochenen Sprachen wird die Intensität der negativ dargestellt Gestalt durch syntaktische Wiederholung wiedergegeben (vgl. Hund, Sohn des Hundes usw.).

Das Hauptmerkmal der heroischen Welt ist die Dichotomie. Im Gegensatz zu den Oghusen spiegelte das hässliche Äußere der Ungläubigen deren innere Widerwärtigkeit und ihren dunklen, hässlichen Geist wider.

Der Parallelität dieser visuellen Charakteristik lässt sich sowohl in der Epik als auch in der Geschichte auf Schritt und Tritt wider.

Bei den mittelalterlichen Glauben und Vorstellungen wird alles, was den existierenden Normen und Regeln nicht entspricht, mit der
Sünde, dem Argen und Bösen in Zusammenhang gebracht. (Versuchen die mittelalterlichen Maler z. B. Gestalten aus der sozialen Unterschicht auf die Leinwand zu bringen, so mussten sie diese körperlich behindert und unvollkommen darstellen).

„Sie sahen dort ein unheimliches Ding liegen... Sie umzingeltenes und ein Krieger stieg ab und versetzte dem Ding einen tritt. Bei jedem Tritt schwoll es weiter an. Noch einige Krieger stiegen ab und traten auf das Häufchen ein. Bei jedem Tritt wurde es größer. Auch Arus Kodscha stieg vom Pferd, um ihm eine Tritt zu geben.

Da blieb sein Sohn hängen und das Häufchen. Heraus kam ein Jung mit dem Körper eines Menschen und mit einem Auge mitten auf dem Scheitel"“ [1, p. 99].

Auch im Nibelungenlied, das die Traditionen des mittelalterlichen Ritterepos und der höfischen Dichtung in sich vereint, erfüllt die Darstellung des Porträts der epischen Helden eine bedeutende Funktion im poetischen System.

Einige deutsche Literaturwissenschaftler, insbesondere der Mediävist Andreas Heusler, meinen, dass die höfische Dichtung die Erzählungskunst wenig beeinflusst hat. Das Nibelungenlied ist ein Heldenepos [6].

Die Analyse der Porträttypen im Nibelungenlied zeigt, dass sich in diesem Epos die „Enzyklopädie“ des 12. Jahrhunderts, seine ethnographische Eigenart widerspiegelt. Die Darstellungstypen des Erscheinungsbildes der Gestalten sind mit den Traditionen der alten deutschen [germanischen] Literatur und der Folklore aufs Engste verbunden. Diese Porträtdarstellungen entsprachen den ästhetischen Werten ihres Zeitalters und dem Geschmack des Zuschauers bzw. Zuhörers. Alle Porträtdetails und - darstellungen fließen ins Sujet der Erzählungskunst hinein. Sie bestimmen die Entwicklung des Sujets und werden dadurch zu einem untrennbaren Bestandteil der literarischen Struktur des Werkes.

Das Heldenepos „Das Nibelungenlied“ beginnt mit der Beschreibung der schönen Kriemhild, der Prinzessin von Burgundenland. Das Lob an Kriemhild verläuft in typischer Form für höfische Dichtung des Mittelalters.

Es wuchs in Burgonden ein schönes Mägdelein,

Wie in allen landen nichts schöners mochte sein.

Kriemhild war sie geheißen und war ein schönes Weib,

Um das viel Degen mussten verlieren Leben und Leib [2, p. 5].

Die Darstellung des Porträts von Siegfried, dem niederländischen Prinzen, der alle Merkmale des höfischen Rittertums verkörpert, rührt von der höfischen Tradition her. Das Porträt von Siegfried ist mit einer märchenhaften Aura umgeben. Er besitzt außergewöhnliche Kraft, ein magisches Schwert und einen unsichtbar machenden Tarnmantel.

Über Brunhild lesen wir: Auf der anderen Seite der See lebte eine schöne Frau, die mit keiner anderen zu vergleichen war. Sie war schön und besaß übernatürliche Kräfte.

Diejenigen, die um sie werben versuchten, wurden verrückt. Gunther, der edle Ritter aus der Region Rhein, vernahm Brunhilds Schönheit von Weitem und verliebte sich in sie.

Es war eine Königstöchter gesessen überm Meer,

Ihr zu vergleichen war keine andre mehr.

Schön war sie aus der Maßen, gar groß war ihre Kraft;

Sie schoss mit schnellen Degen um ihre Minne den Schaft.

Im „Buch des Dede Korkut“ kommen gleiche Situationen vor: Kantuarli, der junge oghusiche Herr begibt sich nach Trapezunt [Trabzon], nachdem er von der Schönheit und Tapferkeit von Seld- 
schan gehört hat: „Nun hatte der König von Trapezunt eine schöne, liebliche Tochter. Sie wusste mit einem Doppelbogen zu schießen, gleichzeitig nach rechts und nach links, und die Pfeife, die sie abschoss, fielen nicht auf den Boden zurück. Das Mädchen besaß drei Bestien, als Brautschatz und Aussteuer“.

Im Nibelungenlied erfüllt das literarische Porträt außerdem noch die Funktion eines bestimmen Sujets.

Die Porträtdarstellung wird abhängig von den Sujet-Situationen vorgestellt. Das entspricht dem Wesen des Heldenepos. Um Siegfried als starken Recken darzustellen, versucht der Verfasser des Nibelungenliedes rituelle Akte, wie den Trachtwechsel, die Aufrüstung und Kriegsvorbereitung in die epische Erzählkunst einzufügen. Die Porträtdarstellungen treten als ein untrennbarer Bestandteil solcher Szenen auf. Selbst in Szenen entgegen den Anstandsregeln finden sich Porträtdarstellungen.

Man hatte Herbergen den Gästen nun genommen.

Der Bote war in reichen Kleidern angekommen

Mit seinen Heergesellen, als sie zu Hofe ritten.

Sie trugen gute Kleider, die waren zierlich geschnitten.

Der unbekannte Spielmann im Nibelungenlied zieht Vergleiche heran, um die Darstellung des Erscheinungsbildes des Helden hervorzuheben. Diese Vergleiche sind in der Regel der Welt der Natur entnommen und werden mit den Regeln der Folklore zum Ausdruck gebracht.

In einigen Fällen versucht der Verfasser des Nibelungenliedes die äußere Schönheit des Helden mit den traditionellen Formen darzustellen:

Sie vergleicht sich an der Schöne wohl der Frauen mein,

Helke, der reichen: Nicht schöner könnte sein

Auf der weiten erde eine Königin:

Wen sie erwählt zum Freunde, der mag wohl trösten seinen Sinn.

Es wuchs in Burgonden ein schönes Mägdelein,

Wie in allen Landen nichts schöners mochte sein.

Im Nibelungenlied nimmt die Darstellung der weiblichen Schönheit eine wichtige Rolle ein. Die Szenen, wie sich die burgundlichen Frauen trachten, sind mit die wichtigen Elemente der Porträt-Ästhetik:

Da ward aus den Schreinen gesucht gut Gewand,

so viel man in der Lade des edeln Staates fand,

Von Borten und von Spangen: des lag genug bereit.

Da zierte sich gar ritterlich manche waidliche Maid [2, p. 36].

Der psychologische Zustand von Kriemhild wird mit den literarischen Details sehr meisterhaft dargestellt:

Dass ihnen Leid hier sprieße, das Herz tats ihnen kund.

Sie mussten alle weinen, was reden mocht ein Mund.

Das Gold vor ihren Brüsten ward von Tränen fahl:

Die fielen ihnen dichte von den Augen zu Tal [2, p. 47].

Nicht nur die Gesichtszüge der Helden interessiert den Verfassern sondern auch ihre treueren Kleider, Kriegsausrüstungen und Pferde:

Die goldfarbenen Zäume führten sie an der Hand;

Der Brustriem war von Seide: So kamen sie ins Land.

Bei der Darstellung der Bekleidung spielt die Symbolik der Farben eine große Rolle. Hierbei wird das Gold als traditionelles Symbol für die Macht verwendet:

Viel goldroter Sättel führten sie ins Land;

Zierlich Schilde und herrlich Gewand

Brachten sie zum Rheine bei dem Hofgelag:

Mancher Ungesunde der Freunde von neuem pflag.
Oder:

Ihre lichten Panzer, die wurden auch bereit

Und ihre festen Helme, ihre Schilde schön und breit [2, p. 25].

Am siebenten Morgen zu Wormes an dem Strand

Ritten schon die Kühnen: da war all ihr Gewand

Aus rotem Gold gewoben, ihr Reitzeug wohlgetan [2, p. 13].

Zusammenfassend kann man behaupten, dass in beiden Epen die Porträttypen mit der Poetik der Folklore zusammenhängen.

Die Forschungsarbeiten belegen, dass eine Klassifizierung und kontrastive Typologie der alten türkischen und germanischen literarischen Porträttypen nicht vorhanden sind. Die literarischen Porträttypen wurden bis jetzt nicht systematisiert, obwohl in einigen Forschungsarbeiten Versuche unternommen wurden, sie zu differenzieren.

\section{Literatur:}

1. Zeynalov F., Olizadə S. Kitabi Dədə-Qorqud. Yazıçı, Baku, 1988.

2. Korneev J. (Übersetzer). Pesn o Nibelungach. Nauka, Leningradş, 1972.

3. Hacıyev T. Dədə Qorqud: dilimiz, düşüncəmiz. Elm, Baku, 1999.

4. Cəmşidov Ş. Kitabi-Dədə Qorqud: tarixi, coğrafi, teksttoloji tədqiq və Drezden əlyazmasının dürüstləşdirilmiş elmi mətni. Elm, Baku, 1999.

5. Abdulla K. Sirr içində dastan və yaxud gizli Dədə Qorqud-2. Elm, Baku, 1999.

6. Heusler A. Deutsche Versgeschichte. 3 Bde. De Gruyter, Berlin, 1929.

7. Chojsler A. Germaniskij geroiceskij epos i skazanie o nibelungach. Moskau, 1960.

Rasulov R. The "Book of the Korkut" and the "Nibelungslied": presentation of literary portraits

Summary. In the German and Turkish literature of the Middle Ages, the methods of human representation and their principles play an important role in research on poetic systems. The authors of the Middle Ages focused on the methods of depicting the portrait, which varied according to the genre.

The "Book of Dede Korkut" and the "Nibelungenlied" analyze complex and complex portraits on a typological and comparative level. Our research can be divided into five portrait groups based on the following criteria:

1. The degree of generalization of the representation (single and group portrait)

2. The subject of the presentation: Part of the appearance of man is represented.

3. The presentation method of the literary figure: portrayed portrait, portrait - gender equality, portrait - comparison etc.

4. The degree of etiquette of the presentation.

5. Character of introduction of a portrait in literary action.

In the epic "Book of Dede Korkut" the method of literary presentation, the portrait method, plays an important role.

In the structure of the epic text are summarized "portrait texts" that are formed by the epithet. In addition to the virtues of the epic heroes in the "portrait texts" are the psychology of their character, their appearance and war symbols (horse, armor, weapon) represented. These portraits also determine the position of the Oghuz nobility (Beys) in the social hierarchy:

Kiyan Seldschuck's son Deli Dundar, who had hit the gate at the pass with the steel gate that made heroes scream with the tip of his sixty-span lance.

Kara Göne's son Kara Budak, who had devastated the fortresses of Diyarbekir and Mardin, who had spit blood with the steel bow from King Kipchak, who had conquered Kazan's daughter with great courage, praising the white-bearded old man among the Oghuz, wherever she praised him Although he was still a young man, dressed in a blouse of red silk, his horse wore an amulet of the hair of a jack-tail around his neck.

Key words: epic, plot, hero, drama, "The Book Dede Korkut" and the "Nibelungenlied". 